BULL. AUSTRAL. MATH. SOC.

MOS 4650,4655

VOL. $2(1970), 253-260$.

\title{
Identities in tensor products of Banach algebras
}

\author{
R. J. Loy
}

\begin{abstract}
Let $A_{1}, A_{2}$ be Banach algebras, $A_{1} \otimes A_{2}$ their algebraic tensor product over the complex field. If $\|\cdot\|_{\alpha}$ is an algebra norm on $A_{1} \otimes A_{2}$ we write $A_{1} \otimes_{\alpha} A_{2}$ for the $\|\cdot\|_{\alpha}$-completion of $A_{1} \otimes A_{2}$. In this note we study the existence of identities and approximate identities in $A_{1} \otimes_{\alpha} A_{2}$ versus their existence in $A_{1}$ and $A_{2}$. Some of the results obtained are already known, but our method of proof appears new, though it is quite elementary.
\end{abstract}

\section{Preliminaries}

The four results collected here are probably already known in one form or another; the proofs are included for completeness.

PROPOSITION 1. Let $A$ be a Bconach algebra, $\left\{e_{\lambda}\right\},\left\{f_{\mu}\right\}$ nets in $A$ such that $\left\|e_{\lambda} x-x\right\| \rightarrow 0,\left\|x f_{\mu}-x\right\| \rightarrow 0$ uniformly on the unit vall of $A$. Then $A$ has an identity.

Proof. Take $s \in\left\{e_{\lambda}\right\}$ such that $\|s x-x\| \leq \frac{1}{2}$ for $\|x\| \leq 1$. Then $s$ is not a left topological divisor of zero, for otherwise there would be $\left\{x_{n}\right\} \subseteq A,\left\|x_{n}\right\|=1$, with $s x_{n} \rightarrow 0$, contradicting $\left\|s x_{n}-x_{n}\right\| \leq \frac{1}{2}$ for each $n$. Similarly there is $t \in A$ which is not a right topological divisor of zero.

Received 10 January 1970. 
Now for each positive integer $n$ take $e_{n} \in\left\{e_{\lambda}\right\}, f_{n} \in\left\{f_{\mu}\right\}$ such that $\left\|e_{n} x-x\right\| \leq \frac{1}{n}, \quad\left\|x f_{n}-x\right\| \leq \frac{1}{n}$ for $\|x\| \leq 1$. Then certainly $\left\|\left(e_{n}-e_{m}\right) t\right\| \leq\left(\frac{1}{n}+\frac{1}{m}\right)\|t\|,\left\|s\left(f_{n}-f_{m}\right)\right\| \leq\left(\frac{1}{n}+\frac{1}{m}\right)\|s\|$ and so, by the choice of $s$ and $t,\left\{e_{n}\right\},\left\{f_{n}\right\}$ are Cauchy, and so converge to elements $e, f \in A$. But then $e$ and $f$ are respectively left and right identities for $A$, and so $e=f$ is an identity.

We will also require the following modification of Proposition 1 . As

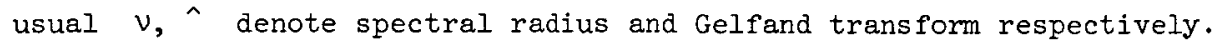

PROPOSITION 2. Let $A$ be a commutative Banach algebra, $\left\{e_{\lambda}\right\} a$ net in $A$ such that $\nu\left(e_{\lambda} x-x\right) \rightarrow 0$ uniformly for $v(x) \leq 1$. Then there is an idempotent $e \in A$ with $\hat{e} \equiv 1$.

Proof. By the same argument as in Proposition 1 there is a sequence $\left\{e_{n}\right\} \subseteq A$ with $\nu\left(e_{n} x-x\right) \rightarrow 0$ uniformly for $\nu(x) \leq 1$, and $\nu\left(e_{n}-e_{m}\right) \rightarrow 0$. It follows that $\left\{\hat{e}_{n}\right\}$ converges uniformly to the constant function $I$ on the carrier space of $A$, which is thus compact. Also, for $n$ sufficiently large $\hat{e}_{n}$ is bounded away from zero, and the elementary argument of [5], pp. 171-2 now furnishes the desired idempotent $e$.

PROPOSITION 3. Let $A$ be a Banach algebra which does not consist entirely of right (Zeft) topological divisors of zero. If $A$ has a left (right) approximate identity $\left\{d_{0}\right\}$ then it has a bounded left (right) approximate identity. Indeed, if $A$ is commutative and $\left\{d_{0}\right\}$ is countable, then $\left\{d_{\rho}\right\}$ is bounded.

Proof. Let $F$ be the family of all finite subsets of $A$, and define a directed set $\Lambda=\{(F, \delta \mathcal{L}: F \in F, I>\delta>0\}$ where $\left(F_{1}, \delta_{1}\right) \leq\left(F_{2}, \delta_{2}\right)$ if $F_{1} \leq F_{2}$ and $\delta_{2} \leq \delta_{1}$. Take $z \in A$ not a right topological divisor of zero. Then for $\lambda=(F, \delta) \in \Lambda$, take $e_{\lambda} \in\left\{d_{p}\right\}$ such that $\left\|e_{\lambda} y-y\right\|<\delta$ for $y \in F \cup\{z\}$, so that if $x \in A, 1>\varepsilon>0$, $\left\|e_{\lambda} x-x\right\|<\varepsilon$ provided $\lambda \geq(\{x\}, \varepsilon)$. It follows that $\left\{e_{\lambda}\right\}$ is a left approximate identity in $A$. Also, $\left\|e_{\lambda} z\right\|<1+\|z\|$ for all $\lambda$, so 
that, by the choice of $z,\left\{e_{\lambda}\right\}$ is bounded.

The last statement is proved in [6], p. 279.

REMARK. The converse is false: $L^{1}(0,1)$ is a radical algebra under convolution, so that all elements are topological divisors of zero, but has a (countable) bounded approximate identity.

Now let $U_{1}, U_{2}$ be seminormed spaces under $p_{1}, p_{2}$ respectively. Corresponding to the normed case define seminorms $p_{\gamma}, p_{\lambda}$ on $U_{1} \otimes U_{2}$ by

$$
\begin{gathered}
p_{\gamma}(x)=\inf \left\{\sum p_{1}\left(u_{i}\right) p_{2}\left(v_{i}\right): x=\sum u_{i} \otimes v_{i}\right\}, \\
p_{\lambda}(x)=\sup \left\{\left|\sum \varphi_{1}\left(u_{i}\right) \varphi_{2}\left(v_{i}\right)\right|: x=\sum u_{i} \otimes v_{i}, \varphi_{j} \in U_{j}^{*},\left\|\varphi_{j}\right\|_{p_{j}}=1,\right. \\
j=1,2\} .
\end{gathered}
$$

As in the normed case $p_{\gamma}$ is the greatest seminorm $p$ on $U_{1} \otimes U_{2}$ such that $p(u \otimes v)=p_{1}(u) p_{2}(v), u \in U_{1}, v \in U_{2} ;$ and so in particular $p_{\lambda} \leq p_{\gamma}$. Using the terminology of [1] a seminorm $p$ on $U_{1} \otimes U_{2}$ will be called admissible if there are positive constants $m, M$ such that $m p_{\lambda} \leq p \leq M p_{\gamma}$.

PROPOSITION 4. Let $U_{j}, p_{j}, j=1,2$ be as above, and $p$ an admissible seminorm on $U_{1} \otimes U_{2}$, with $m$ as above. If $x=\sum_{i=1}^{n} u_{i} \otimes v_{i} \in U_{1} \otimes U_{2}$ with $\left\{v_{i}\right\} \quad$ (or $\left.\left\{u_{i}\right\}\right)$ Linearly independent, then $p_{1}\left(u_{i}\right) p_{2}\left(v_{i}\right) \leq \frac{1}{m} p(x), i=1,2, \ldots, n$.

Proof. Take $I \leq i \leq n$. If $p_{1}\left(u_{i}\right) p_{2}\left(v_{i}\right)=0$ the result is immediate for this $i$, otherwise the Hahn-Banach theorem furnishes $\varphi_{j} \in U_{j}^{*}, j=1,2$ such that $\left\|\varphi_{j}\right\|_{p_{j}}=1, \varphi_{2}\left(v_{k}\right)=\delta_{i k} p_{2}\left(v_{k}\right)$, $\varphi_{1}\left(u_{i}\right)=p_{1}\left(u_{i}\right)$. But then for $w=\sum s_{k} \otimes t_{k} \in U_{1} \otimes U_{2}$, $\varphi_{1} \otimes \varphi_{2}(w)=\sum \varphi_{1}\left(s_{k}\right) \varphi_{2}\left(t_{k}\right) \leq p_{\lambda}(w) \leq \frac{1}{m^{p}}(w)$, and so $\varphi_{1} \otimes \varphi_{2}(x)=p_{1}\left(u_{i}\right) p_{2}\left(v_{i}\right) \leq \frac{1}{m} p(x)$. 


\section{The general (non-commutative) case}

For the remainder of this paper $A_{j}$ will denote a Banach algebra with norm $\|\cdot\|_{j}$, spectral radius $v_{j}, j=1,2 ;\|\cdot\|_{\alpha}$ will be an algebra norm on $A_{1} \otimes A_{2}$ with spectral radius $\nu_{\alpha}$. If $A$ is commutative its carrier space, with the Gelfand topology, will be denoted $\Phi_{A}$.

THEOREM 1. Let $\|\cdot\|_{\alpha}$ be con admissible algebra norm on $A_{1} \otimes A_{2}$. Then $A_{1} \otimes_{\alpha} A_{2}$ has an identity $\iota$ if and only if $A_{1}, A_{2}$ have identities $e, f$, and $\iota=e \otimes f$.

Proof. Suppose that $A_{1} \otimes_{\alpha} A_{2}$ has an identity $\iota$, the converse being immediate. Let $\varepsilon>0$, and take $x=x_{\varepsilon} \in A_{1} \otimes A_{2}$ with $\|x-\imath\|_{\alpha}<\varepsilon$. Then if $x=\sum u_{i} \otimes v_{i}$ and $\|s \otimes t\|_{\alpha} \leq 1$,

$$
\left\|\sum u_{i} s \otimes v_{i} t-s \otimes t\right\|_{\alpha}<\varepsilon, \quad\left\|\sum s u_{i} \otimes t v_{i}-s \otimes t\right\|_{\alpha}<\varepsilon .
$$

Now let $\sum u_{j}^{\prime} s \otimes v_{j}^{\prime}, \sum s u_{k}^{\prime \prime} \otimes v_{k}^{\prime \prime}$ be alternative expressions for $\sum u_{i} s \otimes v_{i} t, \sum s u_{i} \otimes t v_{i}$ respectively, where $v_{1}^{\prime}=v_{1}^{\prime \prime}=t$ and $\left\{v_{j}^{\prime}\right\}$, $\left\{v_{k}^{\prime \prime}\right\}$ are linearly independent sets. Then

$$
\left\|\left(u_{1}^{\prime} s-s\right) \otimes t+\sum_{j \geq 2} u_{j}^{\prime} s \otimes v_{j}^{\prime}\right\|_{\alpha}<\varepsilon, \quad\left\|\left(s u_{1}^{\prime \prime}-s\right) \otimes t+\sum_{k \geq 2} s u_{k}^{\prime \prime} \otimes v_{k}^{\prime \prime}\right\|_{\alpha}<\varepsilon,
$$

and so by Proposition $4\left\|u u_{1}^{\prime} s-s\right\|_{1}\|t\|_{2}<\frac{\varepsilon}{m},\left\|s u_{1}^{\prime \prime}-s\right\|_{1}\|t\|_{2}<\frac{\varepsilon}{m}$, where $m\|\cdot\|_{\lambda} \leq\|\cdot\|_{\alpha} \leq M\|\cdot\|_{\gamma}$. Now $u_{1}^{j}, u_{1}^{\prime}$ depend on $t$ only, not upon $s$, and so, noting that if $\|s\|_{1} \leq 1$ then $\|s \otimes t\|_{\alpha} \leq M\|t\|_{2}$, it follows that $\left\|u_{1}^{\prime} s-s\right\|_{1} \leq \frac{M}{m} \varepsilon,\left\|s u_{1}^{\prime}-s\right\|_{1} \leq \frac{M}{m}$, for $\|s\|_{1} \leq I$. Taking $\varepsilon=1, \frac{1}{2}, \ldots, \frac{1}{n}, \ldots$ we thus obtain sequences $\left\{e_{n}\right\},\left\{e_{n}^{\prime}\right\} \subseteq A_{1}$. such such that $\left\|e_{n} s-s\right\|_{1} \rightarrow 0,\left\|s e_{n}^{\prime}-s\right\|_{1} \rightarrow 0$ uniformly on the unit ball of $A_{1}$, so that by Proposition $1 A_{1}$ has an identity $e$. Similarly $A_{2}$ has an identity $f$, whence $e \otimes f$ is an identity on 
$A_{1} \otimes_{\alpha} A_{2}$ and so must equal $c$.

By an equally simple argument we have the following.

THEOREM 2. Let $\|\cdot\|_{\alpha}$ be an admissible algebra norm on $A_{1} \otimes A_{2}$. If $A_{1}$ and $A_{2}$ each possess a bounded left (right) approximate identity then so does $A_{1} \otimes_{\alpha} A_{2}$. Conversely, if $A_{1} \otimes_{\alpha} A_{2}$ has a left (right) approximate identity then so do $A_{1}$ and $A_{2}$.

Proof. Let $m\|\cdot\|_{\lambda} \leq\|\cdot\|_{\alpha} \leq M\|\cdot\|_{\gamma}$, and take $\left\{e_{\lambda}\right\},\left\{f_{\mu}\right\}$ bounded left approximate identities in $A_{1}$ and $A_{2}$ respectively, with sup $\left\|e_{\lambda}\right\|_{1} \leq C, \quad \sup \left\|f_{\mu}\right\|_{2} \leq C$ for some $C$. Then the set $\left\{e_{\lambda} \otimes f_{\mu}\right\} \subseteq A_{1} \otimes A_{2}$ is bounded, sup $\left\|e_{\lambda} \otimes f_{\mu}\right\|_{\alpha} \leq M C^{2}$, and with the product direction is a left approximate identity in $A_{1} \otimes A_{2}$ (under $\left.\|\cdot\|_{\alpha}\right)$. Since $A_{1} \otimes A_{2}$ is dense in $A_{1} \otimes_{\alpha} A_{2}$ it follows easily that $\left\{e_{\lambda} \otimes f_{\mu}\right\}$ is a bounded left approximate identity in $A_{1} \otimes_{\alpha} A_{2}$.

Conversely, let $\left\{d_{\rho}\right\}$ be a left approximate identity in $A_{1} \otimes_{\alpha} A_{2}$. Let $F$ be a finite subset of $A_{1}, K=\max \left\{\|s\|_{1}: s \in F\right\}+1, \delta>0$, and take $t \in A_{2},\|t\|_{2}=1$. Choose $x \in\left\{d_{p}\right\}$ such that $\|x(s \otimes t)-s \otimes t\|_{\alpha}<\frac{\delta m}{2 M}, s \in F$, and then take $\sum u_{i} \otimes v_{i} \in A_{1} \otimes A_{2}$ with $\left\|x-\sum u_{i} \otimes v_{i}\right\|_{\alpha}<\frac{\delta m}{2 K M^{2}}$. But then $\left\|\sum u_{i} s \otimes v_{i} t-s \otimes t\right\|_{\alpha}<\frac{\delta m}{M}$ for all $s \in F$. Proceeding as in Theorem 1 it follows that there is $u \in A_{1}$ with $\|u s-s\|_{1}<\delta$ for $s \in F$. Now proceed as in Proposition 3 , but without the element $z$, to obtain a net $\left\{e_{\lambda}\right\}$, consisting of such $u$, which is a left approximate identity in $A_{1}$.

Similarly $A_{2}$ has a left approximate identity.

REMARK. The first half of this result appears known, it is used implicitly in [4], Theorem 2.2. The present author has been unable to determine whether addition of the hypothesis of boundedness of $\left\{d_{\rho}\right\}$ in the converse half would ensure boundedness of the resulting nets $\left\{e_{\lambda}\right\}$, $\left\{f_{\mu}\right\}$ in $A_{1}, A_{2}$ respectively. However, if $A_{1}$ and $A_{2}$ are commutative 
and $\left\{\vec{a}_{\rho}\right\}$ is countable and unbounded then not both $\left\{e_{\lambda}\right\},\left\{f_{\mu}\right\}$ are. bounded, for otherwise $\left\{e_{\lambda} \otimes f_{\mu}\right\}$ is a bounded approximate identity in $A_{1} \otimes_{\alpha} A_{2}$, contradicting [6], p. 279. In the general case, if $A_{1}$ and $A_{2}$ do not consist entirely of right (left) topological divisors of zero then Proposition 3 shows that they have bounded left (right) approximate identities, and hence so does $A_{1} \otimes_{\alpha} A_{2}$. Thus if $A_{1}$ and $A_{2}$ are commutative and $\left\{d_{\rho}\right\}$ is countable, then $\left\{d_{p}\right\}$ is bounded.

\section{The commutative case}

The first result concerning identities in $A_{1} \otimes_{\alpha} A_{2}$ was that of Gelbaum [2], Theorem 4, who considered the case $A_{1}, A_{2}$ commutative semisimple, and $\|\cdot\|_{\alpha}=\|\cdot\|_{\gamma}$. This case is included in Theorem 1 above. Recently Lardy and Lindberg [3] have defined an algebra norm $\|\cdot\|_{\alpha}$ on $A_{1} \otimes A_{2}$ to be a spectral tensor norm in the case $v_{\alpha}(u \otimes v)=v_{1}(u) v_{2}(v)$, $u \in A_{1}, v \in A_{2}$. They showed that the natural map of $\Phi_{A_{1}} \otimes_{\alpha} A_{2}$ into $\Phi_{A_{1}} \times \Phi_{A_{2}}$ is surjective if and only if $\|\cdot\|_{\alpha}$ is spectral, and in this case $A_{1} \otimes_{\alpha} A_{2}$ has an identity if and only if $A_{1}$ and $A_{2}$ have identities. In this section we obtain an elementary proof of this result.

LEMMA. Let $A_{1}, A_{2}$ be commutative Banach azgebras, $\|\cdot\|_{\alpha}$ an algebra norm on $A_{1} \otimes A_{2}$. Then $\|\cdot\|_{\alpha}$ is a spectral tensor norm if and only if $v_{\alpha}$ is an admissible seminorm on $A_{1} \otimes A_{2}$, taking the seminorms $v_{1}, v_{2}$ on $A_{1}, A_{2}$. Indeed, $\|\cdot\|_{\alpha}$ is spectral if and only if $\nu_{\alpha}=\nu_{\lambda}$.

Proof. Suppose $\|\cdot\|_{\alpha}$ is spectral. Then by [3], Theorem 1, every multiplicative linear functional on $A_{1} \otimes A_{2}$ is $\|\cdot\|_{\alpha}$-continuous, and so if $x \in A_{1} \otimes A_{2}$,

$$
\begin{aligned}
\nu_{\alpha}(x) & =\sup \left\{|\phi(x)|: \varphi \in\left(A_{1} \otimes A_{2}\right)^{*}, \emptyset \text { multiplicative }\right\} \\
& =\sup \left\{|\varphi \otimes \psi(x)|:(\varphi, \psi) \in \Phi_{A_{1}} \times \Phi_{A_{2}}\right\} \\
& \leq \nu_{\lambda}(x) .
\end{aligned}
$$


Now let $x=\sum u_{i} \otimes v_{i}$, and take $\varepsilon>0$. Then there are $\varphi_{j} \in A_{j}^{*}$, $\left\|\varphi_{j}\right\|_{v_{j}}=1, j=1,2$, with $v_{\lambda}(x) \leq\left|\sum \varphi_{1}\left(u_{i}\right) \varphi_{2}\left(v_{i}\right)\right|+\varepsilon$. Since $\left|\varphi_{1}(u)\right| \leq v_{1}(u), u \in A_{1}$, there is $\varphi_{1}^{\prime} \in \Phi_{A_{1}}$ with $\left|\varphi_{1}^{\prime}\left(\sum u_{i} \varphi_{2}\left(v_{i}\right)\right)\right| \geq\left|\varphi_{1}\left(\sum u_{i} \varphi_{2}\left(v_{i}\right)\right)\right|$ so that

$\nu_{\lambda}(x) \leq\left|\sum \varphi_{1}^{\prime}\left(u_{i}\right) \varphi_{2}\left(v_{i}\right)\right|+\varepsilon$. Similarly, there is $\varphi_{2}^{\prime} \in \Phi_{A_{2}}$ with $\nu_{\lambda}(x) \leq\left|\sum \varphi_{1}^{\prime}\left(u_{i}\right) \varphi_{2}^{\prime}\left(v_{i}\right)\right|+\varepsilon \leq \nu_{\alpha}(x)+\varepsilon$. Since $\varepsilon>0$ is arbitrary it follows that $\nu_{\lambda}(x) \leq \nu_{\alpha}(x)$. Thus $\nu_{\alpha}=\nu_{\lambda}$ and so $\nu_{\alpha}$ is certainly admissible.

Suppose conversely that $\nu_{\alpha}$ is admissible, with $m \nu_{\lambda} \leq \nu_{\alpha}$, and that $\|\cdot\|_{\alpha}$ is not spectral. Since $\nu_{\alpha}(u \otimes v) \leq v_{1}(u) v_{2}(v)$ for all $u \in A_{1}$, $v \in A_{2}$, there must be $u, v$ with $\nu_{\alpha}(u \otimes v) \leq k \nu_{1}(u) \nu_{2}(v)$ for some $k$, $0<k<1$. But then

$v_{\alpha}\left(u^{n} \otimes v^{n}\right) \leq k^{n} v_{1}\left(u^{n}\right) v_{2}\left(v^{n}\right)=k^{n} v_{\lambda}\left(u^{n} \otimes v^{n}\right) \leq \frac{k^{n}}{m} v_{\alpha}\left(u^{n} \otimes v^{n}\right)$, which is impossible for $n$ sufficiently large. Thus $\|\cdot\|_{\alpha}$ is spectral, and so by the above $v_{\alpha}=v_{\lambda}$.

THEOREM 3 (Lardy and Lindberg). Let $A_{1}, A_{2}$ be commutative Banach algebras, $\|\cdot\|_{\alpha}$ an algebra norm on $A_{1} \otimes A_{2}$. If $\|\cdot\|_{\alpha}$ is a spectral tensor norm then $A_{1} \otimes_{\alpha} A_{2}$ has an identity if and only if $A_{1}$ and $A_{2}$ have identities.

Proof. Suppose $A_{1} \otimes_{\alpha} A_{2}$ has an identity, the converse being immediate. Arguing as in Theorem 1 , but using $\nu_{1}, v_{2}, \nu_{\alpha}$ in place of $\|\cdot\|_{1},\|\cdot\|_{2}$ and $\|\cdot\|_{\alpha}$, there is $\left\{e_{n}\right\} \subseteq A_{1}$ with $v_{1}\left(e_{n} s-s\right) \rightarrow 0$ uniformly for $v_{1}(s) \leq 1$. But then by Proposition 2 there is an idempotent $e \in A_{l}$ with $\hat{e} \equiv \mathcal{I}$. Similarly there is an idempotent $f \in A_{2}$ with $\hat{f} \equiv 1$. The result now follows as in [3], Theorem 4 . 


\section{References}

[1] H.R. Fischer, "Über eine Klasse topologischer Tensorprodukte", Math. Ann. 150 (1963), 242-258.

[2] B.R. Gelbaum, "Tensor products and related questions", Trans. Amer. Math. Soc. 103 (1962), 525-548.

[3] L.J. Lardy and J.A. Lindberg Jr, "On maximal regular ideals and identities in the tensor product of commutative Banach algebras", Canad. J. Math. 21 (1969), 639-647.

[4] Kjeld B. Laursen, "Ideal structure in generalized group algebras", Pacific J. Math. 30 (1969), 155-174.

[5] Charles E. Rickart, General theory of Bcnach algebras (Van Nostrand, Princeton, New Jersey, 1960).

[6] C. Robert Warner and Robert Whitley, "A characterization of regular maximal ideals", Pacific J. Math. 30 (1969), 277-281.

Carleton University, O+tawa, Canada. 